\title{
Any Data, Any Time, Anywhere: Global Data Access for Science
}

\author{
Kenneth Bloom ${ }^{\dagger}$, Tommaso Boccali ${ }^{\uparrow}$, Brian Bockelman*, Daniel Bradley ${ }^{\ddagger}$, Sridhara Dasu ${ }^{\ddagger}$, \\ Jeff Dost ${ }^{\S}$, Federica Fanzago $\|$, Igor Sfiligoi ${ }^{\S}$, Alja Mrak Tadel ${ }^{\S}$, Matevz Tadel ${ }^{\S}$, \\ Carl Vuosalo ${ }^{\ddagger}$, Frank Würthwein ${ }^{\S}$, Avi Yagil ${ }^{\S}$ and Marian Zvada* \\ *Department of Computer Science and Engineering, University of Nebraska-Lincoln \\ ${ }^{\dagger}$ Department of Physics and Astronomy, University of Nebraska-Lincoln \\ ${ }^{\ddagger}$ Department of Physics, University of Wisconsin-Madison \\ $\S$ Department of Physics, University of California, San Diego \\ IIstituto Nazionale di Fisica Nucleare (INFN), Sezione di Pisa \\ "Istituto Nazionale di Fisica Nucleare (INFN), Sezione di Padova
}

\begin{abstract}
Data access is key to science driven by distributed high-throughput computing (DHTC), an essential technology for many major research projects such as High Energy Physics (HEP) experiments. However, achieving efficient data access becomes quite difficult when many independent storage sites are involved because users are burdened with learning the intricacies of accessing each system and keeping careful track of data location. We present an alternate approach: the Any Data, Any Time, Anywhere infrastructure. Combining several existing software products, AAA presents a global, unified view of storage systems - a "data federation," a global filesystem for software delivery, and a workflow management system. We present how one HEP experiment, the Compact Muon Solenoid (CMS), is utilizing the AAA infrastructure and some simple performance metrics.
\end{abstract}

\section{Introduction}

Scientific research in our time is increasingly driven by large datasets, which in coming years will approach the exabyte scale. In the current era of computing, these data may be distributed across a large number of geographically separated computing facilities. A fundamental problem for data-driven scientific research enabled by distributed highthroughput computing (DHTC) is how to give scientific communities fast and efficient access to the data to ensure that the progress of science is never slowed by computational infrastructure.

Experiments conducted at the Large Hadron Collider (LHC), the proton collider operated by CERN in Geneva, Switzerland, are exemplars of data-driven scientific research on a grand scale. The experiments are prodigious producers of data, with each major experiment generating tens of petabytes of data each year. These data must be stored, processed and then analyzed by the thousands of scientists participating in the experiment. As a whole, each experimental collaboration measures thousands of quantities that are documented by hundreds of scientific papers, which must be completed in a timely fashion.
It has been a challenge to achieve these goals. The scientists are distributed around the world, as are the highthroughput computational facilities Originally, the facilities were organized in a tiered structure, with the Tier- 0 facility at CERN, Tier-1s at national facilities, Tier-2s at major institutions and Tier-3s at most collaborating institutions. Each tier had well-defined roles in computation to satisfy LHC needs. However, the tiered structure often placed impediments to easy data access. The provisioning of computing and storage resources varied significantly in spite of the seemingly hierarchical structure. Every computing site only hosted a subset of the data, and the sites were typically accessed through grid infrastructures. Without a locationindependent data access technology, processing jobs requiring a certain subset of the data as input had to be executed at the same site where that subset was housed.

This need to co-locate the storage systems that host datasets and the processors that analyze them created a fundamental inefficiency in the processing system. Processors tended to be free at sites which had the least popular data, while sites hosting data that were simultaneously analyzed by many scientists had their processors oversubscribed. Content Delivery Networks (CDNs) approach this problem by automatically redistributing data based on access patterns; however, a blind caching-based approach is not feasible for our purpose due to the working set size being larger than any single data center. Centers that hosted no data at all but had significant processing power were difficult to use effectively. Many physicists had processors available at their home institutions, but did not have the expertise to operate large storage systems, restricting their ability to analyze data. Data access was not merely a matter of volume: If the only way to access data is through the batch system, users incurred a huge latency penalty when trying to debug their code over even a few megabytes of data.

Thus, a desire to blur the distinction among different tiers of computing facilities turned out to be desirable and was encouraged, primarily due to the availability of highbandwidth wide-area network (WAN) connections among all tiers. However, careful attention to latency and other issues 
due to reliance on WAN needed to be addressed explicitly.

In 2010, we began to solve this problem for the Compact Muon Solenoid (CMS) experiment by building an international-scale data access infrastructure under the name "Any Data, Anytime, Anywhere" (AAA) that would remove the requirement of co-location of storage and processing resources. The infrastructure is transparent, in that users have the same experience whether the data they analyze is halfway around the world or in the room next door. It is reliable, in that end users rarely see a failure of data access when they run their application. It enables greater access to the data, in that users no longer have the burden of purchasing and operating complex disk systems. In fact, any data can be accessed anytime from anywhere with an internet connection.

This AAA system is an example solution for the more general problem of large-scale access to distributed scientific data. With its emphasis on reliable and transparent access, easy integration with applications, and excellent efficiency, it is a system that can be used for a wide variety of "big data" scientific problems that are solved across DHTC systems. As we move into an era of gigantic datasets, commercial cloud centers with billions of processors and content delivery networks, researchers across many scientific domains will want to make use of any data, anytime, anywhere.

In this paper, we describe the AAA infrastructure. Section 2 describes the underlying technology choices and their implementations. Section 3 discusses the use cases for the CMS experiment and how they have improved scientific productivity. Section 4 documents system performance metrics. Section 5 describes how the AAA technology is starting to be used in other scientific communities beyond experimental particle physics. Section 6 concludes with thoughts on the future of distributed data access.

\section{AAA Technologies}

The AAA infrastructure is a unique synthesis of preexisting software commonly used by the DHTC community. We have taken the following and adopted them to the use case at hand:

- AAA data federation: Based on XRootD [1], this system provides uniform remote data access to all of CMS's on-disk data.

- Workflow management software: HTCondor [2] provides workflow management and glideinWMS provides computing resource provisioning.

- Global file systems: To distribute CMS software, we utilize CernVM File System (CVMFS) [3] and have integrated it with Parrot [4] to emulate it on hosts where it is otherwise unavailable.

\subsection{XRootD Data Federation}

As in Ref. [5], we define a data federation to be a collection of disparate storage resources managed by cooperating but independent administrative domains transparently accessible via a common namespace. To users, interaction with a data federation is akin to accessing popular services like DropBox rather than managing individual filesystems across dozens of storage sites. For storage sites, our implementation is an overlay of the existing site storage: an important characteristic, as each site is run by an independent team of administrators, with different sites not always sharing the same goals.

The XRootD software [1] is used to create the AAA data federation [5]. The data federation serves the CMS global namespace via a tree of XRootD servers as depicted in Figure 1. The leaves of this tree are referred to as data sources, as they serve data from local storage systems. Each storage system is independent of the others, allowing for a broad range of implementations and groups to participate in the federation as long as they expose an agreed-upon namespace through the XRootD software. The non-leaf nodes in Figure 1 have no storage, but may redirect client applications to a subscribed data source that has the requested file. Each host is subscribed to at most one redirector, called a manager; loops are disallowed. If the requested file is not present on a server subscribed to the redirector, then the client will be redirected to the current host's manager. The manager continues the process until either a source is found or the client is at the root of the tree. An application may thus be redirected to any host in the federation, irrespective of the branch point it initially accesses.

Each redirector maintains a cache of file location information and a negative cache of files with no source. When a client requests a file, the redirector first checks the caches for potential location information. If the source is not known and there has been no recent failed lookup, the redirector sends a multicast request to all of its branches and leaves. If the redirector determines no source has the requested file, then the client is sent to the redirector's manager. File location (or non-existence) is cached by each redirector, avoiding multicast queries for common files. As data sources are composed of independently-run storage systems, each has a different availability schedule (according to the local sites maintenance schedule or outages); if the client is sent to a non-functioning source, it will return to the previous redirector requesting a different source. Resilience against poor data sources is explored further in Section 2.7 .

The redirector file discovery and client data access are done with distinct protocols: the former is the cmsd (Cluster Management Service) protocol and the latter xrootd. As the client does not need to understand the cmsd protocol, the data access protocol is completely independent; for clients, HTTP is also implemented and HTTP 2.0 is under investigation. In addition to providing basic file access, the XRootD protocol allows for pluggable authentication and authorization systems; this has allowed CMS to re-utilize its existing X-509-based infrastructure [6] without having to change the XRootD protocol. 


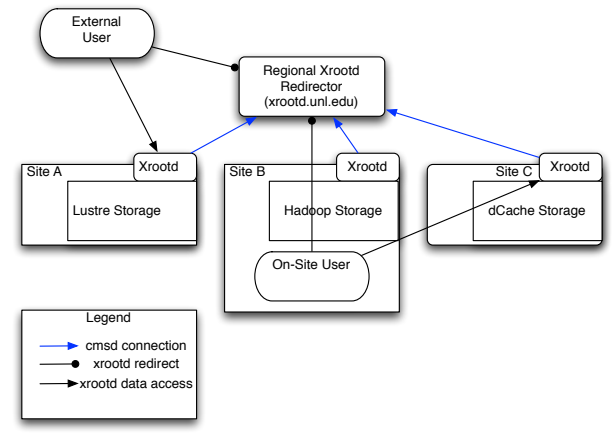

Figure 1. An overview of the production site topology for the AAA data federation.

\subsection{CMS Global Federation}

Using the XRootD federation mechanisms described above, we have implemented a global data access federation for CMS. The implemented topology has a threelayer XRootD hierarchy: the global redirector, two regional redirectors (one in the US and one in Europe), and at least one data source per site. The hierarchy may be deeper: some sites run their own local XRootD federation, invisible to the global one, for scalability reasons. The middle layer of regional redirectors are organized to mitigate the impact of wide-area network latency since application performance drops significantly when latency is above $50 \mathrm{~ms}$. When possible, clients are configured to first contact their respective regional redirector. This step mitigates query propagation; for example, a client contacting the US redirector for a file will only trigger file location queries for US sites. European sites will only be queried if the file is not located in the US. See Figure 1 for an overview of the production topology.

Organizing the federation's hierarchy around network regions is one technique for improving observed performance: It reduces the average access latency for applications. Another performance technique is to maintain consistently high-quality data sources within the federation. Accordingly, we have split the set of data sources into production and transitional; the latter are sites which do not meet minimum performance guarantees. Previously, in Figure 11, we described only the production sites; Figure 2 shows a more complete global federation picture, including the transitional sites. The global federation will first try to serve data from a site within the production federation; if a file does not exist in the production federation, the client will be redirected to the transitional federation. This process will cause the low-performance sites to be used only if no other source is available, regardless of network latency. Transitional sites are not held to the same standard of performance of their storage as the production sites that are allowed to be part of the main branch of the global data federation. To be in the production federation, a site must be a Tier- 1 or Tier- 2 site, and thus a member of the Worldwide LHC Computing Grid (WLCG) [7] with a commitment to certain levels of facility availability, and meet minimal performance criteria that are described in Section 4

Altogether, the global data federation has provided many benefits to CMS, as described in Section 3

\subsection{XRootD Proxy Cache}

Despite significant investments to reduce application latency sensitivity, some use cases still see significant benefits from caching data locally at the processing site. This may be due to limited WAN bandwidth, sites that run specific processing workflows that have known working set sizes, or the use of unoptimized applications.

Accordingly, we have extended XRootD's proxying functionality to be a caching proxy (similar in approach to a HTTP proxy); all client requests go to the proxy host which, in turn, acts as a client of the AAA data federation. Unlike a HTTP proxy, the average file size in XRootD is quite large - typically over one gigabyte - and XRootD clients tend to read out non-sequential subsets of the file. Popular HTTP caches will read in the full request to disk before returning any data to the client; here, sub-file caching is needed to avoid significant delays. The caching proxy will initially read only the byte ranges requested by the application; as the application progresses, the caching proxy will read from data sources only the byte ranges requested not already cached locally. Depending on the configuration, the proxy may concurrently read the remaining missing portions of the file to the local storage. The proxy cache is discussed further in Ref. [8].

\subsection{XRootD Monitoring}

Monitoring plays several important roles in any XRootD data federation. Remote data access provides more complex failure modes: The number of clients accessing the storage is no longer limited to the number of local CPUs, storage system performance is more visible outside the site, and clients running on a local host may access any of dozens of data centers around the globe. To manage this complexity, the AAA infrastructure has significant investments in monitoring.

On a basic level, our monitoring provides basic service health checks. Is the service accessible over the Internet? Are users able to authenticate? Will it redirect clients and serve data? These checks are achieved by a set of status probes directed at each site in the federation. A second level of monitoring gathers data about the state and operations of individual servers. The most important monitored quantities are the outgoing data rate, number of connected clients, rates of new connections, and authentication successes or failures. Several low-level performance indicators from XRootD servers are also available, making it possible to trace usage of system resources. This data is stored in a time-series database used for evaluation of site performance and for understanding of performance issues.

The first two monitoring levels cover operational aspects of the infrastructure for serving data but give no details about individual file accesses. The third level of 


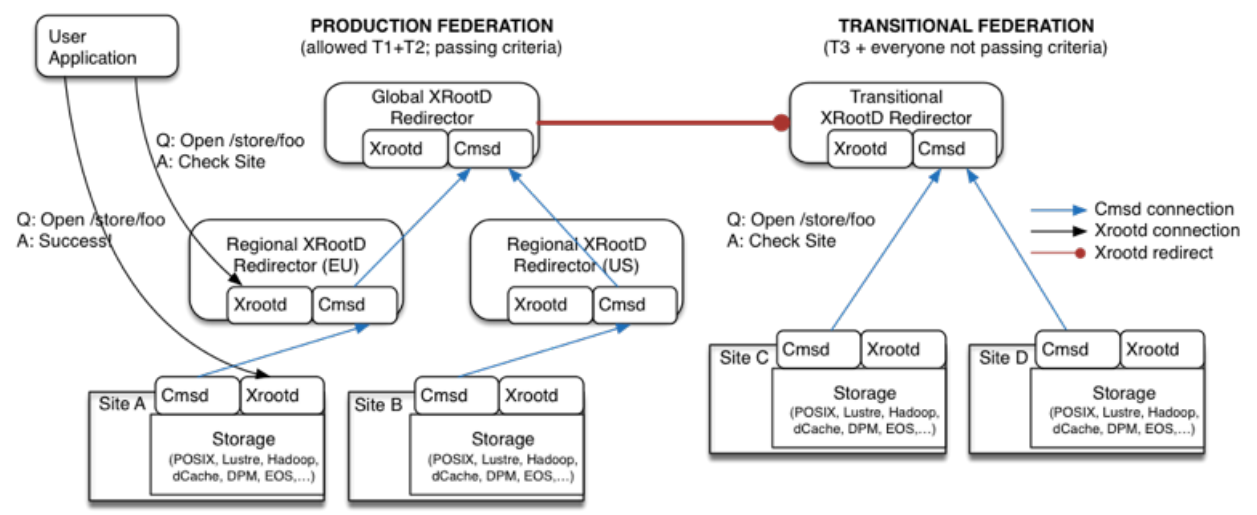

Figure 2. An overview of the global AAA data federation as deployed for CMS.

monitoring collects details about individual user sessions including complete user and file information, file open and close times, and amount of transferred data. It is possible to include details about individual read requests made by clients thus allowing in-depth analysis of file-access patterns of client applications. The results of such analyses are used to identify parts of application's I/O stack that require improvement, to determine the types of applications that are best suited for remote data-access, and for tuning of cachingproxy operational parameters. The user session information includes client identifiers, allowing us to correlate the I/O performance with the user job performance.

\subsection{HTCondor and glideinWMS}

The resource provisioning and workload submission infrastructure in CMS is built based on HTCondor [2] and glideinWMS [9]. CMS tools for data analysis, simulation, and reconstruction submit workloads into an HTCondor system that implements a "global pool" of CMS resources. Based on the number of idle jobs within the HTCondor pool, glideinWMS provisions resources across compute and storage clusters worldwide. Resources include clusters "owned" by CMS within the WLCG, allocations at supercomputing centers, and cloud-based resources. This provisioning is done by submitting a "glidein" to the clusters via various grid [10] and cloud interfaces (such as Amazon EC2), or even just SSH [11]. Upon starting on the resource, the glidein joins the global pool, and multiple jobs are sched- uled in parallel or in series on the resource, depending on available hardware.

The existence of the global pool gives CMS central control over how jobs are prioritized and assigned to individual sites. As a late-binding system, it allows for significant flexibility. This feature is put to use in conjunction with AAA in job overflows, as described in Section 3.2

\subsection{CVMFS and HTTP proxies}

CVMFS [3] is used to establish a uniform runtime environment for CMS applications. CVMFS in turn uses Squid [12] caches for local caching of the libraries required by the applications. The combination of CVMFS and Squid caches provides access to all active versions of the CMS application software without having to explicitly install any of them locally. Instead, libraries are pulled and cached as needed. These features can be used to straightforwardly add additional resources into the global pool, even if those resources do not have any affiliation with CMS that would lead them to have particular software and services available in advance.

\subsection{Multisource Client}

A difficulty in setting up a data federation such as AAA lies in data source selection: Given a client file request, to which data source should the central host redirect the client? If the redirector does not have the potential source 
locations cached, it must broadcast a query to all sources to discover potential locations. How long should it wait for a response? If two potential sources have been discovered, should the redirector wait an additional second for a third, better source?

There are significant penalties for incorrect source selection. Despite efforts to reduce the impact of latency and bandwidth of CMS applications, if a transatlantic source is selected when a nearby one is available, there is significant application performance penalty to this apparent "misredirection". Worse yet - optimal redirection is not possible because the source optimal at redirection time may possibly degrade after the client begins to read data.

To solve the issues of mis-redirection and uneven source performance, we developed the multisource client. This client, covered at length in Ref. [13], will maintain connections to multiple data sources. Each read request is divided proportional to recent source performance. Further, the client will randomly probe for additional, faster sources while the file remains open. These steps improve uneven performance by reading proportionally less data from slower sources and dropping the slowest if a significantly faster one is found. The random additional source probe allows the client to find alternate sources in case it was mis-redirected.

\section{CMS Use Cases}

The technologies described in Section 2 allow for the access of remote data in CMS applications simply by naming a remote data file as input and specifying that it be accessed through an XRootD redirector. In this section, we give examples of how CMS has used this functionality in many different ways to improve processing reliability and efficiency and to give a better experience for individual end users and improve throughput for a wide variety of processing tasks at many different scales.

\subsection{Fallback Access}

Many of the CMS use cases rely on the so-called fallback mechanism. While this mechanism was first conceived as a way to protect processing jobs against local storage failures, it can also be used to transparently access remote data under a wide variety of conditions.

In the CMS software (CMSSW) framework, the location of input files within the storage system are determined through the Trivial File Catalog (TFC). The TFC maps the logical file names that identify files within the CMS data catalog to the physical file names for the file replicas at a given computing site. Unique to each site, the TFC is described by a small number of XML files.

The TFC can be configured to specify a secondary choice of physical file name should the primary choice fail to open for whatever reason. The secondary choice can be set to be the logical filename but accessed through the redirector rather than a local file. This allows an automatic fallback in case of an error: Should there be any sort of fault in the local storage system, the user's CMSSW application immediately retries the file opening through the AAA infrastructure, without any additional action by the user. In doing so, the processing is much more robust against local filesystem problems, allowing for greater job-completion efficiency with less user intervention. In addition, it provides functionality that many other use cases can built upon; e.g., applications can deliberately ignore data locality because when they try to access a file locally via the TFC, they get automatically redirected to a remote fileserver that has the file when it isnt available locally.

\subsection{Overflows}

As mentioned above, the original CMS computing model was based on the principle that applications were allowed to process only local files. This restriction led to inefficiencies where sites with data of limited interest would tend to have spare processing capacity while sites hosting heavily used data would be oversubscribed. To level out these inefficiencies, CMS operates a "global queue" across all sites worldwide using HTCondor via glideinWMS. When one site within a region is oversubscribed, glideins at other sites within the region can accept applications destined for the oversubscribed site. When the application starts and tries to open a file that does not exist locally, it thus triggers the fallback mechanism described above. It falls back to the regional redirector, is redirected to another server that has the file, and reads from this remote server. A maximum number of such overflow jobs can be configured in the global pool for each site to avoid overloading the storage at the source site.

\subsection{Small Computing Sites}

AAA has made it possible to conveniently instantiate "super small Tier-3" (SST3) environments that are fully integrated into the global system of Tier-2 and Tier-1 sites. A SST3 will have an XRootD proxy cache, a regular XRootd server serving local disk, an HTCondor mini-cluster, and CVMFS. All of these services are managed from one of the Tier-2s via Puppet [14]. The HTCondor mini-cluster then overflows into the CMS global pool, implementing a "submit locally - compute globally" concept. The proxy cache pulls in files from the global CMS data federation that are locally accessed, and the regular XRootD server allows the "SST3 owners" to analyze their private files even when their jobs overflow into the global infrastructure.

An SST3 can be as small as a single node or may be an extended mini-cluster of a few nodes. A single SST3 node may also function as CMS-specific infrastructure co-located with a larger cluster operated for the entire university by its IT division. In that case, overflow may be configured to preferentially target the local campus cluster rather than the global CMS system.

Such a system makes its local data available in the federation. An even simpler SST3 would not host any data of its own, but simply read data from other sites in the federation as necessary. HTCondor is used for bulk processing jobs 
that are submitted from this computer to run elsewhere on grid resources. Interactive work running on the SST3 uses XRootD to access data files from anywhere in the CMS data federation, including the output files from their bulk processing jobs, which are typically in Tier- 2 storage. Here, too, the system configuration can be managed from another cluster via Puppet, and the environment and software can be provided through CVMFS, allowing for low administrative overhead. Such a system could be deployed and maintained by a few graduate students, allowing university research groups to have easy access to computing with no additional staff support.

\subsection{World-wide Production Processing}

The CMS computing model includes the notion of a distributed tape archive across the Tier- 1 centers. Each dataset is assigned to a custodial Tier-1. The custodial Tier-1 is responsible to guarantee long term archiving of the dataset on tape, as well as any future primary re-processing of it. This model implies that the throughput with which data at a custodial Tier-1 can be processed is limited by the total processing capacity of the Tier-1. Experience has shown that this arrangement is a limiting factor during large-scale (re-)processing campaigns.

With AAA, any CPU at any site can, in principle, be used to process any data from any of the custodial Tier1s. In practice, AAA has enabled processing of data at Fermilab from CPUs at NSF supercomputers like Gordon at the San Diego Supercomputing Center (SDSC), processing at Fermilab of data that is custodial at another Tier-1, and processing across several Tier- $2 \mathrm{~s}$ of data at a custodial Tier-1. In other words, the overall production processing has become much more flexible, providing overall more throughput globally.

In the future, we envision production processing across Tier-1s, some subset of the Tier-2s, allocations at NSF or DOE supercomputers, opportunistic resources on the Open Science Grid [15], and commercial clouds.

\subsection{Storage Healing}

Several of the Tier-2s in the United States have adopted HDFS [16] as their local storage infrastructure because of its excellent scalability and easy, robust operations. HDFS can be FUSE-mounted [17] from worker nodes of the local cluster to give the appearance of a global filesystem across all disks at the site. HDFS allows the specification of a replica count on a per-file and per-directory tree basis. By default, all Tier-2s have set the replica count to two for all files in order to safeguard against disk failures, with the result that the usable space at one of the HDFS based Tier-2s is slightly less than half the raw disk space at the site.

To decrease the average replica count at a particular site to below two, while maintaining robustness against disk failures, we used the XRootD proxy cache technology described in Section 2.3 to implement robust, self-healing storage without full replication. The idea is simple. We intercept any read errors from HDFS in the FUSE mount, and redirect reading of those missing or corrupt blocks to an XRootD proxy cache. The proxy cache then fetches the missing bytes via the XRootD data federation from a remote site. An independent daemon then lazily fixes up the corrupt or missing files in HDFS by replacing the missing blocks, and relinking the files. The net result is that applications experience a small slowdown in reading when they hit a missing block, but the FUSE-mounted HDFS system never fails to deliver any file blocks, even if the disks that contained them have died long ago.

In practice, the Tier- $2 \mathrm{~s}$ that have deployed this functionality now decide by dataset about the replica count for the files in that dataset. This decision is driven entirely by the desire for access redundancy to support aggregate read performance rather than robustness.

\section{Performance and Usage}

AAA has performed successfully in supporting remote data access needs of the CMS experiment over the last two years. Individual sites serving data have been tested and validated to ensure that they can handle anticipated maximum loads. While sites are first tested to verify that they have sufficient performance to join the production federation, they are also re-tested regularly (approximately once per week) to check that their infrastructure continues to work at sufficient scale. Tests are run using a dedicated HTCondor pool, and the results are used to provide feedback to site administrators on system performance.

The performance tests probe both file opening and file reading and are designed to push the conceivable limits of the system. Across the entire CMS distributed computing system, there can be as many as 100,000 jobs running simultaneously. A given job typically opens files at a rate of $0.001 \mathrm{~Hz}$. Sites are thus tested to a file-opening rate of up to $200 \mathrm{~Hz}$, the equivalent of 200,000 jobs trying to open files at a single site. While this is well above the rate that would be expected at any single site, it is a test of the robustness of the full XRootD infrastructure, which multi-casts file location queries to all sites. A typical CMS job reads files at a rate of $0.25 \mathrm{MB} / \mathrm{s}$. The file-reading tests probe reading rates up to a load eqivalent to 4000 jobs, or $1 \mathrm{~GB} / \mathrm{s}$. The requirements for joining the production federation are much smaller than the bounds of performance testing; for inclusion, sites are required to demonstrate opening rates of at least $10 \mathrm{~Hz}$ and reading rates equivalent to 600 simultaneous jobs, or $150 \mathrm{MB} / \mathrm{s}$.

Figures 3 and 4 show test results for the largest single site in the CMS distributed computing system, which is hosted by Fermilab. This site performs extremely well.

The AAA federation is used extensively within CMS. Figure 5 shows the daily usage of the federation over the course of a recent month. On any given day, the average throughput of the system averages $1 \mathrm{~GB} / \mathrm{s}$, with tens of different sites being used as sources. These source sites vary greatly in the amount of data hosted and are distributed 


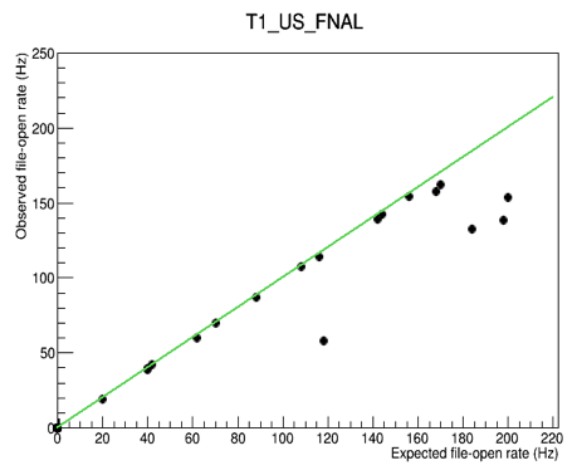

Figure 3. File-opening performance for remote jobs reading files at Fermilab via AAA. The green line represents targeted performance, where the observed opening rate matches the expected rate. The plot shows that Fermilab can far exceed the target rate of $10 \mathrm{~Hz}$. The points off the line come from jobs slowly terminating at the end of the test.

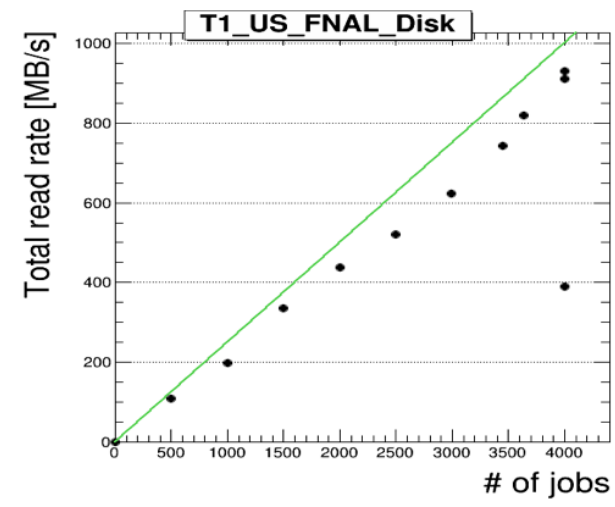

Figure 4. Remote reading read rate of files stored at Fermilab via AAA. The green line represents the target rate of $0.25 \mathrm{MB} / \mathrm{s}$ per job, with the Fermilab data points fairly closely following the line. The site far exceeds the minimum necessary rate of 600 jobs making simultaneous reads.

around the world. This widespread participation in the federation is evidence for its success within CMS.

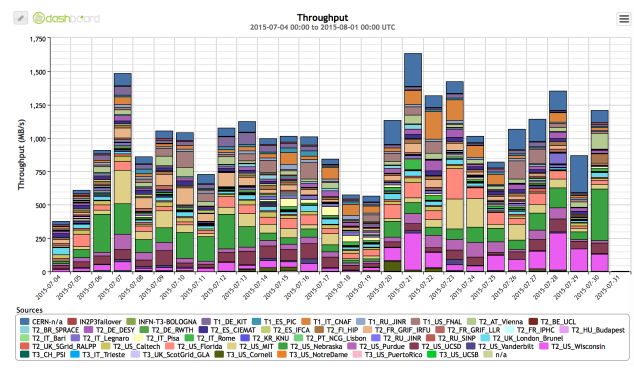

Figure 5. Daily average read rate from the CMS AAA data federation over a one month period. Each color in the histogram represents a different source site as indicated in the legend. The plot captures all sites that have deployed the detailed monitoring system described in Section 2.4

\section{AAA In Other Communities}

In this paper we have described a number of concepts and how they have been applied to support the science of the CMS Collaboration at the LHC. The needs of CMS drove the development of the integrated concepts to a significant degree. An additional goal has been to generalize these tools for use in other scientific communities. We have started collaboration with the Open Science Grid (OSG), SDSC and the California Institute for Telecommunications and Information Technology (Calit2). We expect there to be substantial research and development challenges, and thus intellectual opportunities in these generalizations. For example, we have focused our tuning efforts to applications based on the ROOT framework [18] and protocol; how essential is the concept to this application? Even within other science communities beyond experimental particle physics that use ROOT, one should expect a wider diversity of applications with wider sensitivity to latency. For the proxy caches outlined in Section 2.3, it is not clear how to optimally determine the needed hardware configuration. Calit2 has developed cost-effective "Flash I/O Network Appliances" (FIONA) that they have deployed across a large number of institutions across the West Coast of the United States, while the OSG deployment in Section 5.1 is based on SATA disks. The FIONAs are engineered for maximum network capability per cost, while the OSG StashCache focuses on disk space per cost as most important metric. These examples are just some of the R\&D challenges we expect to be facing as we explore these future opportunities. In the following, we briefly describe these two projects.

\subsection{StashCache: Distributing User Data on the Open Science Grid}

The Open Science Grid provides access to clusters at universities and national laboratories in the U.S. and Latin America for any science domain. During 2014, roughly 800 million hours across 200 million jobs were provided by 67 clusters. The number of hours per cluster ranged from one hour to 100 million hours per year. Two thirds of this was consumed by the LHC experiments ATLAS, ALICE, and 


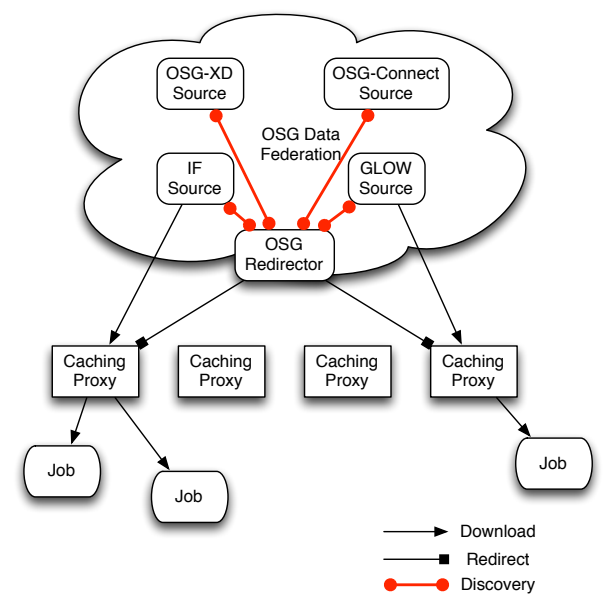

Figure 6. The StashCache data access infrastructure.

CMS. The remaining third went to 18 other high energy and nuclear physics experiments (13\%), various life sciences $(10 \%)$, and a wide range of other sciences (11\%), including social sciences such as economics. Among these research groups, only the LHC community was in a position to perform data analysis at significant scale. All others were limited to datasets no more than a few GB in size.

To increase the capabilities of science other than the LHC from the gigabyte scale to the terabyte scale, OSG is deploying the AAA proxy cache technology described in Section 2.3 to provide a distributed cache across the U.S. with multiple "origin servers" as entry points for adding data into the federation. Figure 6 depicts this architecture. As we have less control over the science applications in OSG compared to CMS, we have preferred the proxy-cache approach to hide latency and collaborate with users on working set size to avoid cache thrashing.

The goal of this project is to support all OSG users who have common input data files, with an initial goal of handling up to a few terabyte-scale datasets. Users will upload copies of their data to pre-defined entry points (sources) and will set up jobs to fetch data without having to understand implementation architecture and details or initial file location. Internally, XRootD will copy files from the entry points to OSG caches that are located at or near OSG sites where user jobs run. So that applications need not know about the XRootD protocol, OSG sets up the runtime environment through the use of preload libraries [19] that intercept POSIX system calls and redirect them with the XRootD client. The StashCache data federation intends to correlate HTCondor-based monitoring of jobs with the server side XRootD monitoring using the functionality introduced by the work described here.

\subsection{The Pacific Research Platform and LHC@UC}

The San Diego Supercomputer Center, in collaboration with the California Institute of Telecommunications and
Information Technologies have chosen our technologies as part of the Pacific Research Platform (PRP). PRP is a system that spans 20 universities, colleges, and national laboratories on the West Coast. It connects the individual science DMZs [20] into a regional science network. To make such a regional network infrastructure useful to science requires higher-level services for data and compute access. The technologies described here were chosen as initial deployment targets, and the LHC user community distributed across six University of California campuses are among the science users of these technologies on the PRP. Any scientist at these institutions can submit workloads locally that access data from the federation via the local cache deployed inside the high-performance regional network. In addition, jobs can overflow from the local cluster across clusters in the PRP. Jobs then access the data they need from anywhere in the PRP via the data federation. Conceptually, this implements a distributed Tier-3 infrastructure for the ATLAS and CMS institutions participating in the PRP, and also serves as a implementation model for other science communities.

\section{Conclusion}

We have described an integrated system of computing tools that is now serving the needs of a large, distributed community of scientists who must access a large, distributed dataset. With these technologies, scientists of the CMS experiment are able to access their data more easily and reliably than ever before, which will speed the process of scientific discovery. We have demonstrated that the system can perform at the level needed to meet the goals of the experiment. The tools that we have developed can be straightforwardly applied to other scientific areas that can benefit from distributed high-throughput computing, and some of these applications are already under development. As dataintensive sciences move into the exabyte era, researchers everywhere will be able to use these tools to fully exploit any data, any time, anywhere.

\section{Acknowledgments}

We thank our collaborators on the Compact Muon Solenoid experiment for providing a platform from which the AAA project could be built, and their welcoming acceptance of our efforts. This work is supported in part by the National Science Foundation through awards PHY-1104664, PHY-1104549 and PHY-1104664.

\section{References}

[1] A. Dorigo, P. Elmer, F. Furano, and A. Hanushevsky, "Xrootd-a highly scalable architecture for data access," WSEAS Transactions on Computers, vol. 1, no. 4.3, 2005.

[2] D. Thain, T. Tannenbaum, and M. Livny, "Distributed computing in practice: the condor experience.” vol. 17, no. 2-4, 2005, pp. 323-356.

[3] J. Blomer, P. Buncic, and T. Fuhrmann, "Cernvm-fs: delivering scientific software to globally distributed computing resources," in Proceedings of the first international workshop on Network-aware data management. ACM, 2011, pp. 49-56. 
[4] D. Thain and M. Livny, "Parrot: An application environment for dataintensive computing," Scalable Computing: Practice and Experience, vol. 6, no. 3, pp. 9-18, 2005.

[5] L. Bauerdick, D. Benjamin, K. Bloom, B. Bockelman, D. Bradley, S. Dasu, M. Ernst, R. Gardner, A. Hanushevsky, H. Ito et al., "Using xrootd to federate regional storage," in Journal of Physics: Conference Series, vol. 396, no. 4. IOP Publishing, 2012, p. 042009.

[6] I. Foster and C. Kesselman, "The globus toolkit," 1999, pp. 259-278.

[7] "Worldwide LHC Computing Grid," http://wlcg.web.cern.ch/

[8] L. Bauerdick, K. Bloom, B. Bockelman, D. Bradley, S. Dasu, J. Dost, I. Sfiligoi, A. Tadel, M. Tadel, F. Wuerthwein et al., "Xrootd, diskbased, caching proxy for optimization of data access, data placement and data replication," in Journal of Physics: Conference Series, vol. 513, no. 4. IOP Publishing, 2014, p. 042044.

[9] I. Sfiligoi, D. C. Bradley, B. Holzman, P. Mhashilkar, S. Padhi, and F. Wurthwein, "The Pilot Way to Grid Resources Using glideinWMS," in Proceedings of the 2009 WRI World Congress on Computer Science and Information Engineering - Volume 02, ser. CSIE '09. Washington, DC, USA: IEEE Computer Society, 2009, pp. 428-432. [Online]. Available: http://dx.doi.org/10.1109/ CSIE.2009.950

[10] B. Bockelman, T. Cartwright, J. Frey, E. Fajardo, B. Lin, M. Selmeci, T. Tannenbaum, and M. Zvada, "Commissioning the htcondor-ce for the open science grid," Journal of Physics: Conference Series, p. to appear, 2014.

[11] D. Weitzel, I. Sfiligoi, B. Bockelman, J. Frey, F. Wuerthwein, D. Fraser, and D. Swanson, "Accessing opportunistic resources with bosco," Journal of Physics: Conference Series, vol. 513, no. 3, p. 032105, 2014.

[12] D. Wessels, "Squid: The definitive guide," 2004.

[13] B. Bockelman, "Optimizing high-latency i/o in cmssw," Journal of Physics: Conference Series, vol. 513, no. 4, p. 042006, 2014. [Online]. Available: http://stacks.iop.org/1742-6596/513/i=4/ $\mathrm{a}=042006$

[14] Puppet. (2015) It configuration management. [Online]. Available: https://puppetlabs.com/

[15] R. Pordes, D. Petravick, B. Kramer, D. Olson, M. Livny, A. Roy, P. Avery, K. Blackburn, T. Wenaus, F. Würthwein et al., "The open science grid," in Journal of Physics: Conference Series, vol. 78, no. 1. IOP Publishing, 2007, p. 012057.

[16] B. Bockelman, "Using hadoop as a grid storage element," Journal of Physics: Conference Series, vol. 180, no. 1, p. 012047, 2009. [Online]. Available: http://stacks.iop.org/1742-6596/180/i=1/ $\mathrm{a}=012047$

[17] M. Szeredi et al., "Fuse: Filesystem in userspace," Accessed on, 2010.

[18] R. Brun and F. Rademakers, " $\{$ ROOT $\}$ an object oriented data analysis framework," Nuclear Instruments and Methods in Physics Research Section A: Accelerators, Spectrometers, Detectors and Associated Equipment, vol. 389, no. 12, pp. 81 - 86, 1997, new Computing Techniques in Physics Research V. [Online]. Available: http://www.sciencedirect.com/science/article/pii/ S016890029700048X

[19] XRootd. (2015) Preload library. [Online]. Available: https:// confluence.slac.stanford.edu/display/ds/Xrootd+Preload+Library/

[20] E. Dart, L. Rotman, B. Tierney, M. Hester, and J. Zurawski, "The science dmz," in Proceedings of the International Conference for High Performance Computing, Networking, Storage and Analysis on - SC '13., 2013. 